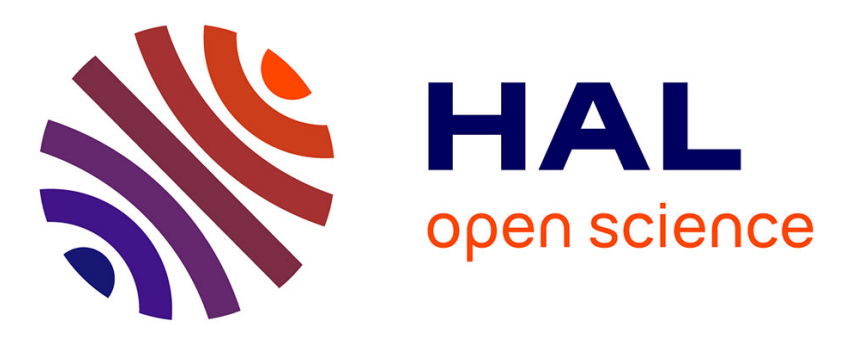

\title{
A hybrid temporal gis representation for coastal dynamics
}

\author{
Younes Hamdani, Rémy Thibaud, Christophe Claramunt
}

\section{To cite this version:}

Younes Hamdani, Rémy Thibaud, Christophe Claramunt. A hybrid temporal gis representation for coastal dynamics. 27th ACM SIGSPATIAL International Conference on Advances in Geographic Information Systems, ACM SIGSPATIAL GIS 2019, Nov 2019, Chicago, United States. pp.584-587, 10.1145/3347146.3359357. hal-02433752

\section{HAL Id: hal-02433752 https://hal.science/hal-02433752}

Submitted on 4 Jun 2021

HAL is a multi-disciplinary open access archive for the deposit and dissemination of scientific research documents, whether they are published or not. The documents may come from teaching and research institutions in France or abroad, or from public or private research centers.
L'archive ouverte pluridisciplinaire HAL, est destinée au dépôt et à la diffusion de documents scientifiques de niveau recherche, publiés ou non, émanant des établissements d'enseignement et de recherche français ou étrangers, des laboratoires publics ou privés. 


\section{A Hybrid Temporal GIS Representation for Coastal Dynamics}

\author{
Younes Hamdani \\ younes.hamdani@ecole-navale.fr \\ Naval Academy Research Institute \\ Brest, France
}

\author{
Rémy Thibaud \\ National School of Advanced \\ Techniques (ENSTA Bretagne) \\ Brest, France \\ remy.thibaud@ensta-bretagne.fr
}

\author{
Christophe Claramunt \\ Naval Academy Research Institute \\ Brest, France \\ christophe.claramunt@ecole- \\ navale.fr
}

\begin{abstract}
The experimental research developed in this paper introduces a hybrid temporal GIS representation for studying the dynamics of a coastal seabed. The approach is based on a dual temporal GIS data model that combines the object and field views of space. The first concept is the one of an object-field defined as a field in which each location is associated to one or more geographic objects type. The second key concept is the field-object defined as an object with internal heterogeneity conceptualized as a field. The main idea behind this approach is to provide a flexible representation that supports field and object evolutions. A series of spatio-temporal queries have been categorized and specified on top of the hybrid representation and shows the interest of the combination of the field and object abstractions at the data manipulation level. The approach has been applied to a coastal data environment, and implemented within the PostgreSQL RDBMS and its spatial extension PostGIS.
\end{abstract}

\section{CCS CONCEPTS}

- Information systems $\rightarrow$ Spatial-temporal systems; Geographic information systems.

\section{KEYWORDS}

Hybrid GIS, Object-Field, Field-Object, Coastal Dynamics

\section{INTRODUCTION}

Since the emergence of GIScience, several authors have successively contributed to the object and field views. Nevertheless, both conceptualizations, while structurally different, offer complementary views that might be combined to offer additional data manipulation capabilities that will be valuable in many environmental application contexts [1]. Several recent works introduced hybrid conceptualizations but either primarily based on the object or field views. A first approach considers an object-field defined as a field in which a location is associated not only with a value, but also with a geographic object type [4]. According to [2], the spatial embedding of objects assigned to a specific location may or may not include this location. A second approach considers a field-object as an object with internal heterogeneity conceptualized as a field. According to [3], a field-object can be seen as a generalization of an object which is qualified by the internal variation of its fields. The experimental research presented in this paper introduces a formal conceptualization of this dual approach with an integration of the temporal dimension, and illustrates its potential by a series of spatiotemporal queries. This is first designed at the formal level, then conceptually using a query scheme where different hybrid queries are categorized. The main idea behind this conceptual and logical approach is to provide a flexible representation that support dual field and object evolution. The whole approach is applied and experimented in the context of the study of the dynamics of a coastal seabed. The rest of the paper is structured as follows. Section 2 introduces the formal background and basic concepts to extend the fieldbased and object-based representations towards the temporal domain. The formalization of the hybrid representation is presented in Section 3. Section 4 illustrates the potential of our modelling approach by introducing a series of query types related to the evolution of a dune phenomenon in a coastal environment. Finally Section 5 draws a conclusion of the paper.

\section{BASICS}

Let us represent space as a dual set of spatial locations and a set of continuants, that is, objects that span over time. Let $L$ 
denote the spatial domain which is defined as set of spatial locations such that $L=\left\{l_{1}, l_{2}, l_{3}, \ldots, l_{n}\right\}$ and $\mathcal{P}(L)$ is the set of all subsets of $L$, and $C$ the set of continuants. Time is represented by the domain $(T,<)$ where $T$ is a set of instants such that $T=\left\{t_{1}, t_{2}, t_{3}, \ldots, t_{n}\right\}$ ordered by the relation $<$. We denote $L \times T$ as set of spatial-temporal locations $(l, t) \in L \times T$.

\subsection{Field-based temporal representation}

Let us define the set of values $V=\left\{v_{1}, v_{2}, v_{3}, \ldots, v_{n}\right\}$ as a collection of sample numerical values that denote a continuous property $m$ like Temperature, Elevation, Tidal current, etc. Formally, a field $f$ is defined as a function $f: L \rightarrow V$ Where $L$ denotes a set of spatial locations.

Several functions could be defined to extend a field $f$ to the temporal domain. Let us first define a function state ${ }_{m}: X \rightarrow$ $V$ that assigns a set of values $V$ to a cross-product of the spatial and temporal domains $X=L \times T$.

Applying the process of currying that transforms a function with more than one parameter into a sequence of functions, each with a single parameter, we obtain the function hist $_{m}: L \rightarrow(T \rightarrow V)$ that assigns a pair time-value to a spatial location. With $m$ that denotes a property, hist $_{m}(l)$ gives a set of composite values $\left(t_{k}, v_{j}\right)$ valid at the location $l$ for that property $m$ (we assume that at any time we have the same fixed sample of locations).

\subsection{Object-based temporal representation}

The object-based representation refers to the concept of continuant which is defined as an object having a type $d$ and that endures over time, undergoes change and preserves its identity. It exists as a whole at each moment of its lifetime. Henceforth we call an object of type $d$ a $d$-Object. Let us define a value domain $H$ as a set of attributes of a $d$-object.

We consider that at each valid time instant $t$, a $d$-Object $c$ is assigned to a value of a domain $H$. Formally this is represented by the function $f_{h}: C \times T \rightarrow H$.

Let us represent the spatial component of an object, formally, by the function $f_{g}: C \times T \rightarrow$ geom, where geom corresponds to the geometry of the $d$-Object which can be a point, line or polygon.

\section{HYBRID SPATIO-TEMPORAL REPRESENTATION}

The objective of the hybrid spatio-temporal representation is to combine the two field and objects views of space. This is first done through the function $f_{l}: C \times T \rightarrow \mathcal{P}(L)$ which represents a subset of locations associated with a given $d$ object at a given time.

This mapping is based on a spatial-temporal rule where:

$$
f_{l}(c, t)=\left\{L_{i} \in \mathcal{P}(L) \mid \forall l \in L_{i} \text { then } l \cap f_{g}(c, t) \neq 0\right\}
$$

The above constraint specifies that at a time $t$ the spatial component of the $d$-object $c$ (i.e., $\left.f_{g}(c, t)\right)$ intersects a location $l$ of $L$. Based on the function $f_{l}$, let us define two key concepts: Object-Field and Field-Object.

\subsection{Dynamic Object-Field}

An Object-Field maps every location to one or more objects (i.e., point, linear, areal). These objects may or may not contain the original field location in their extent.

The Object-Field representation can help to explore, for instance, how the hydrodynamic conditions (i.e., field view), for example the tidal current, might impact the migration velocity of marine dunes (i.e., object view) in a given area. Let us formally define this concept.

Definition 1 : An Object-Field is an ordered pair $\left(d, f_{c}\right)$, where $d$ is an object type and $f_{c}$ is a field whose values range over aggregations of $d$-objects such that $f_{c}: S \times T \rightarrow \mathcal{P}(C)$, where $\mathcal{P}(C)$ denotes the power set of $C$. The subset of $d$-objects, that is related to a specific spatio-temporal location by the function $f_{c}$, is constrained by the following rule:

$$
f_{c}(l, t)=\left\{C_{i} \in \mathcal{P}(C) \mid \forall c \in C_{i} \text { then } l \in f_{l}(c, t)\right\}
$$

This rule specifies that the location $l$ belongs to the subset of locations returned by the function $f_{l}$.

This definition preserves the functional mapping between the dynamic field and $d$-objects. This is particularly relevant when a spatio-temporal location is assigned to more than one d-objects (i.e., case of overlapping objects).

The previous definition covers Nulls, single $d$-object and aggregation of $d$-bjects. A NULL value is represented by the empty set. For a single object, $f_{c}(l, t)$ is restricted to being a singleton set, whereas there is no restriction on the aggregation of $d$-objects, except the rule defined above.

\subsection{Dynamic Field-Object}

The second concept which turns out to be close to the inverse of the above Object-Field $f_{c}$ is called the Field-Object. This consists of objects characterized by internal variations. These are qualified as a field where every location is assigned to a continuous property value. This supports a close mapping from $d$-objects to their respective associated fields.

An illustrative example of this abstraction is given by a bathymetry data set and a an evolving marine dune. While a dune is considered as a moving object with its identity, shape, attributes (e.g., wavelength, height) and relationships with other objects (e.g., a dune is inside a sandbank area), a dune has important internal field-based variations such as elevation and slope. Let us formally define this concept.

Firstly, let us define $A$ as the set of all ordered pair timevalue $(t, v) \in(T, V)$ and $\mathcal{P}(A)$ is the power set of $A$.

Definition 2:A Field-Object is an ordered pair ( $m, f o)$, where $m$ is a specific property (e.g., temperature) and fo is a function that assigns to each d-objectc (e.g., dune) at a time t a subset of spatial locations with their associated historical values related 
to the property $m: f_{o}: C \times T \rightarrow(L \rightarrow \mathcal{P}(A))$. The value fo $\left(c, t_{i}\right)$ is constrained by the following rules:

$$
\begin{aligned}
& f o\left(c, t_{i}\right)=\left\{\left(l, A_{i}\right) \in(L, \mathcal{P}(A)) \mid A_{i} \cdot t \subseteq t_{i}\right\}, \\
& \begin{array}{ll}
\text { i) } l \in f_{l}\left(c, t_{i}\right) & \text { ii) } A_{i} \subseteq H_{i s t}(l)
\end{array}
\end{aligned}
$$

The first condition specifies that the location $l$ belongs to the subset of locations returned by $f_{l}$. The second one ensures that the subset $A_{i}$ belongs to the set of pair time-value returned by $\operatorname{Hist}_{m}(l)$.

\subsection{Hybrid Approach Conceptual Scheme}

Let us apply the hybrid conceptual approach to the characterization of the dynamic of a coastal dune. We based our hybrid query scheme on the framework of Sinton [6] that successively considers location, time, and attribute as fixed, controlled, and measured components. This scheme generates two types of hybrid queries that offer complementary Object-Field or Field-Object constructs to enhance spatiotemporal query capabilities. Each one is categorized as a query type (Table 1$)$.

\begin{tabular}{lll} 
& & Hybrid Queries \\
\cline { 2 - 3 } & Query Type 1 & Query Type 2 \\
\hline Fixing & Property & Object attribute \\
Controlling & location/Time & Time/d-Object \\
Measuring & Object Evolution & Field Evolution \\
Used Approach & Object-Field & Field-Object
\end{tabular}

Table 1: Hybrid conceptual query scheme.

\section{EXPERIMENTATION}

Following the framework suggested in Table 1, the focus is on the study of the interaction between the spatio-temporal variability of the seabed morphology and marine dunes evolution. Understanding of erosion, growth, displacements, and other mechanisms still constitute a scientific challenge for the understanding of marine dune behavior [5].

\subsection{Implementation}

The field based representation of space corresponds to the bathymetry which describes a continuous surface related to the seabed, while the object based representation is related to the extraction of the crestline from the bathymetry in order to define a marine dune as a geometric object. The data set used in our research is given by a set of bathymetric surveys recorded over three years from 2009 to 2011 as Digital Terrain Models (DTM) with $2 \mathrm{~m}$ grid spacing. The study area is located about 10km off the shore of Western Brittany in France in the junction area between the English Channel and the
North Atlantic Ocean. The implementation is based on the open source object-relational database system PostgreSQL, and its spatial extension PostGIS. This extension supports the vector and raster spatial data models, this being a strong requirement of our approach. For the field based view, the focus is on depth property of the seabed. The Postgis raster functions (ST_PixelAsPoints and ST_Value) are used in order to extract a point geometry from each pixel with their related depth values. For the object based view, 21 different dunes have been identified, represented by polygons and then are stored in a specific table. The implementation of the two forms of hybrid representation is applied by developing two Set Returning Functions for the object-field and fieldobject using the procedural language $p l / p g S Q L$. Queries are specified with SQL, and Qgis was used as the geospatial core for visualization.

\subsection{Query Examples}

Let us illustrate the two types of queries applied on top of the hybrid approach in order to explore marine dune dynamics. Three representative examples are given bellow:

Query 1: Return the dunes having an area larger than $7 \mathrm{~km} 2$ and that are impacted by a deposition process higher than $15 \mathrm{~m}$ between two times $t_{1}$ and $t_{2}$. This query is decomposed into two steps as follows:

$$
\begin{aligned}
& Q 1:=\left\{f_{c}(l, t) \mid \operatorname{hist}_{m i}(l)\left(t_{2}\right)-\text { hist }_{m i}(l)\left(t_{1}\right)>15\right\} \\
& Q 2:=\left\{c \in Q 1 \mid \operatorname{area}\left(f_{g}\left(c, t_{i}\right)\right)>7000 \text { and } t_{i} \in\left[t_{1}, t_{2}\right]\right\}
\end{aligned}
$$

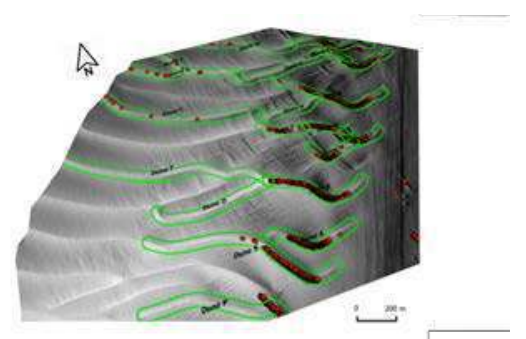

Figure 1: Set of selected dunes (in green) with areas larger than $7 \mathrm{~km} 2$, and deposition higher than $15 \mathrm{~m}$ (located with red dots) between $\mathrm{t} 1$ and $\mathrm{t} 2$.

The query illustrated in Figure 1 returns the set of dunes affected by an increase of elevation higher than 15 meters (i.e., deposition process) somewhere on their surface. The query applies the Object-field function $f_{c}$, where $Q_{1}$ corresponds to the output of $f_{c}(l, t)$ which represents a subsets of object $c$, and where the location $l$ is selected under the condition that its depth difference is higher than 15 meters. The next step applies the instance of $Q 1$ to identify the subset of continuants $c$ that have an area higher than $7 \mathrm{~km}^{2}$. 
Query 2: Where are located the erosion process and its amplitude related to the dune with the most important migration rate between two times $t_{1}$ and $t_{2}$ ? This query is decomposed into three steps as follows: $Q:=\left\{(c, m g) \mid m g=\operatorname{distance}\left(\operatorname{ctr}\left(c, t_{1}\right), \operatorname{ctr}\left(c, t_{2}\right)\right)\right\}$ $R:=\left\{f o\left(Q . c,\left[t_{1}, t_{2}\right]\right) \mid Q . m g=\operatorname{Max}(Q . m g)\right\}$ $W:=\left\{(R . l, v) \mid v<0, \quad v=\left(\right.\right.$ hist $_{m}(R . l)\left(t_{2}\right)-$ hist $\left.\left._{m}(R . l)\left(t_{1}\right)\right)\right\}$

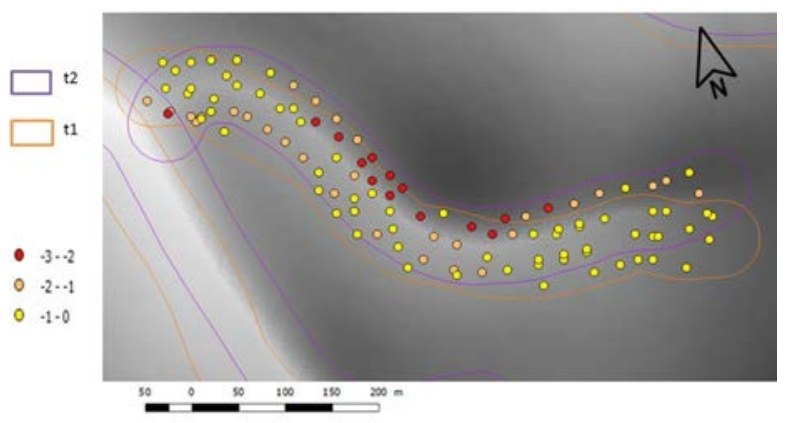

Figure 2: Locations and amplitudes of erosion on the seabed (in meters) for the dune with the most important displacement between $\mathrm{t} 1$ and $\mathrm{t} 2$.

This query, whose output is given in Figure 2, illustrates how the dynamics (i.e., migration) of a given dune might influence changes in the field of seabed depth property. Firstly, the set of dunes with their respective migration rates is selected and the result is assigned to the variable $Q$. That is done by computing the distance between the centroids (i.e., $c t r$ ) of each dune $c$ at two instants $t_{1}$ and $t_{2}$. Secondly, the input of fo is restricted to the dune $c$ having the most important migration rate by applying the function Max on $\mathrm{mg}$ attribute. Therefore, $R$ gets the output of $f o\left(c,\left[t_{1}, t_{2}\right]\right)$ which represents a set of ordered pairs $\left\langle l, \operatorname{hist}_{m}(l)\right\rangle$.

Finally, the variable $W$ gets the result of $R$ and returns a spatial field (i.e., set of ordered pairs $(l, v))$ which denotes the erosion (i.e., negative depth change).

Query 3: what is the seabed elevation change related to the dune $A$ and to the dune that came the closest between $t_{1}$ and $t_{3}$ ?

Let us first define a function $f_{r}$ that computes the distances between a given d-object $c_{A}$ and all its neighborhoods objects $c$ at each time $t_{k}$ :

$f_{r}\left(c_{A}\right)=\left\{\left(c, t_{k}, r\right) \mid c \neq c_{A} \wedge r=\operatorname{distance}\left(f_{g}\left(c_{A}, t_{k}\right), f_{g}\left(c, t_{k}\right)\right)\right\}$

Then the query is decomposed into three steps as follows:

$Q:=\left\{(c, g) \mid c \in f_{r}\left(c_{A}\right) . c \wedge g=f_{r}\left(c_{A}\right)\left(c, t_{3}\right)-f_{r}\left(c_{A}\right)\left(c, t_{1}\right)\right\}$

$R:=\left\{f o\left(c^{\prime},\left[t_{1}, t_{3}\right]\right) \mid c^{\prime}=c_{A} \vee c^{\prime} \in Q . c \wedge Q . g=\operatorname{Min}(Q . g)\right\}$

$W:=\left\{(R . l, v) \mid v<0, \quad v=\left(\right.\right.$ hist $_{m}(R . l)\left(t_{3}\right)-$ hist $\left.\left._{m}(R . l)\left(t_{1}\right)\right)\right\}$

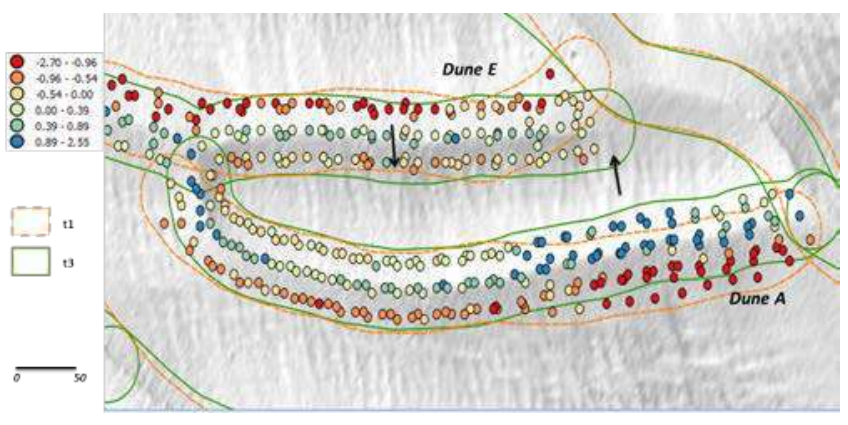

Figure 3: Locations and amplitudes of erosion (red dots) and deposition (blue dots) for the dune $A$ and for the dune that came the closest (Dune $\mathrm{E}$ ) between $\mathrm{t} 1$ and $\mathrm{t} 3$.

The first step computes the magnitude $g$ which assesses the distance rate of decrease or increase between the Dune A and all its neighborhood between two given times. The next step is to select the dune that came the closest to dune A based on $\operatorname{Min}(Q . g)$. The result is assigned to the function fo. As for query 2 , the result gives a spatial field (i.e., set of ordered pairs $(l, v))$ which denotes erosion and deposition trends. The query output is illustrated in Figure 3.

\section{CONCLUSION}

The research introduced in this paper develops a hybrid GIS approach that favors a representation of continuous and discrete geographical properties. We redefine the Object-Field and Field-Object concepts to ensure bi-directional mappings between these two views of space. The aim is to capture some dynamic properties at different levels of abstraction. We develop a conceptual scheme that supports data manipulation capabilities that take advantage of the hybrid approach. The potential of the whole approach is illustrated by a series of spatio-temporal queries types oriented to the seabed and the marine dune dynamic in a coastal environment.

\section{REFERENCES}

[1] Thomas J. Cova. 2016. Data Model, F-Objects and O-Fields. International Encyclopedia of Geography: People, the Earth, Environment and Technology (2016), 1-5.

[2] Thomas J. Cova and Goodchild Mickael F. 2002. Extending geographical representation to include fields of spatial objects. International fournal of Geographical Information Science 16, 6 (2002), 509-532.

[3] Thomas J. Cova, May Yuan, and Goodchild Mickael F. 2007. Towards a general theory of geographic representation in GIS. International fournal of Geographical Information Science 21, 3 (2007), 239-260.

[4] Antony Galton. 2001. A formal theory of objects and fields. In International Conference on Spatial Information Theory : Lecture Notes in Computer Science, Vol. 2205. Springer, Berlin, Heidelberg, 458-473.

[5] Eric Hardin, Helena Mitasova, Laura Tateosian, and al. 2014. GIS-based analysis of coastal Lidar time-series. Springer New York. (book).

[6] David Sinton. 1978. The inherent structure of information as a constraint to analysis: Mapped thematic data as a case study. Harvard Papers on Geographic Information Systems (1978). 